\title{
The evolutionary host switches of Polychromophilus: a multi-gene phylogeny of the bat malaria genus suggests a second invasion of mammals by a haemosporidian parasite
}

\author{
Fardo Witsenburg ${ }^{1 *}$, Nicolas Salamin ${ }^{1,2}$ and Philippe Christe ${ }^{1}$
}

\begin{abstract}
Background: The majority of Haemosporida species infect birds or reptiles, but many important genera, including Plasmodium, infect mammals. Dipteran vectors shared by avian, reptilian and mammalian Haemosporida, suggest multiple invasions of Mammalia during haemosporidian evolution; yet, phylogenetic analyses have detected only a single invasion event. Until now, several important mammal-infecting genera have been absent in these analyses. This study focuses on the evolutionary origin of Polychromophilus, a unique malaria genus that only infects bats (Microchiroptera) and is transmitted by bat flies (Nycteribiidae).

Methods: Two species of Polychromophilus were obtained from wild bats caught in Switzerland. These were molecularly characterized using four genes (asl, clpc, col, cytb) from the three different genomes (nucleus, apicoplast, mitochondrion). These data were then combined with data of 60 taxa of Haemosporida available in GenBank. Bayesian inference, maximum likelihood and a range of rooting methods were used to test specific hypotheses concerning the phylogenetic relationships between Polychromophilus and the other haemosporidian genera.

Results: The Polychromophilus melanipherus and Polychromophilus murinus samples show genetically distinct patterns and group according to species. The Bayesian tree topology suggests that the monophyletic clade of Polychromophilus falls within the avian/saurian clade of Plasmodium and directed hypothesis testing confirms the Plasmodium origin.

Conclusion: Polychromophilus' ancestor was most likely a bird- or reptile-infecting Plasmodium before it switched to bats. The invasion of mammals as hosts has, therefore, not been a unique event in the evolutionary history of Haemosporida, despite the suspected costs of adapting to a new host. This was, moreover, accompanied by a switch in dipteran host.
\end{abstract}

Keywords: Polychromophilus, Malaria, Haemosporida, Chiroptera, Plasmodium, Host switch, Phylogenetic analysis, Outgroup selection

\section{Background}

Five genera belonging to the order of Haemosporida (Apicomplexa) are known to infect mammals: Plasmodium, Hepatocystis, Polychromophilus, Nycteria and Rayella $[1,2]$. The dipteran vectors of the first three haemosporidian genera are represented by Culicidae (Anopheles spp.),

\footnotetext{
* Correspondence: fardo.witsenburg@unil.ch

'Département d'Ecologie et Evolution, Université de Lausanne, Biophore, UNIL-Sorge, 1015 Lausanne, Switzerland

Full list of author information is available at the end of the article
}

Ceratopogonidae and Nycteribiidae respectively, while the vectors of Nycteria and Rayella are unknown [1,2]. Culicidae and Ceratopogonidae also act as vectors of the avian and saurian Haemosporida $[1,3]$. These shared vectors suggest that haemosporidian parasites might have invaded mammals multiple times during their evolution. On the other hand, the switch to mammals is thought to have been an evolutionary demanding process for the parasite [4] and therefore a rare event [5].

\section{C) Biomed Central}


Molecular phylogenetic studies to date have been able to detect only a single host switching event to mammals: mammalian Plasmodium and Hepatocystis, the main mammal-infecting genera, had a common origin and formed a monophyletic sister clade to sauropsid Plasmodium $[6,7]$. However, these phylogenetic studies suffer from incomplete taxon sampling with most investigations including, besides the genera Plasmodium and Hepatocystis, only the avian Haemoproteus and Leucocytozoon. Consequently, with no knowledge of the evolutionary origin of the other mammalian haemosporidian groups (i.e. Rayella, Nycteria, Polychromophilus), a second move into mammals cannot be excluded.

One possible approach for resolving this standing question is to select a haemosporidian genus that could potentially have switched to mammal hosts independently of mammalian Plasmodium/Hepatocystis. A good candidate genus for this is Polychromophilus as it is well described, with the majority of its life cycle well documented, including its vector stage. Moreover, it infects mammals but is not transmitted by Culicidae like Plasmodium, nor Ceratopogonidae like Hepatocystis, but by Nycteribiidae (Diptera: Hippoboscoidea). Furthermore, Polychromophilus' vertebrate host species range is restricted to the insectivorous bats (Microchiroptera). A phylogenetic analysis of Polychromophilus can, therefore, elucidate whether it arose through an independent switch to mammal hosts [8].

Only five species of Polychromophilus are known to exist. While they can be distinguished by their slight differences in ultrastructure, they are mainly classified based on host-type $[9,10]$. Landau et al. [10] proposed dividing the genus into two subgenera based on their gametocyte morphology: 1) the subgenus Polychromophilus, with $P$. (P.) melanipherus as the type species, which has gametocytes similar to the type 'malariae'; 2) the subgenus Bioccala with type species Polychromophilus (B.) murinus whose gametocytes resemble the benign tertian parasites of birds and reptiles (see Figure 1) [10]. Later, it was even suggested that the subgenus Bioccala be raised to genus level [11]; however, this was not reflected in the literature [12]. Moreover, the morphological distinctions between the species have been described as 'slight' [9] and how well they reflect the genetics of the genus has not been studied.

The Nycteribiidae vectors are also known as nycteribids or bat flies. These haematophagous flies are completely adapted to a parasitic lifestyle in the fur of bats in that they have lost their wings, have no or reduced eyes and possess hooking claws which allow them swift movements through the fur $[13,14]$. Coradetti [15] was the first to detect sporozoites in their salivary glands and later studies confirmed his finding $[12,16]$.

When an evolutionary conservation of hosts is assumed, Polychromophilus' unique host-vector combination of Mammalia and Nycteribiidae gives rise to two

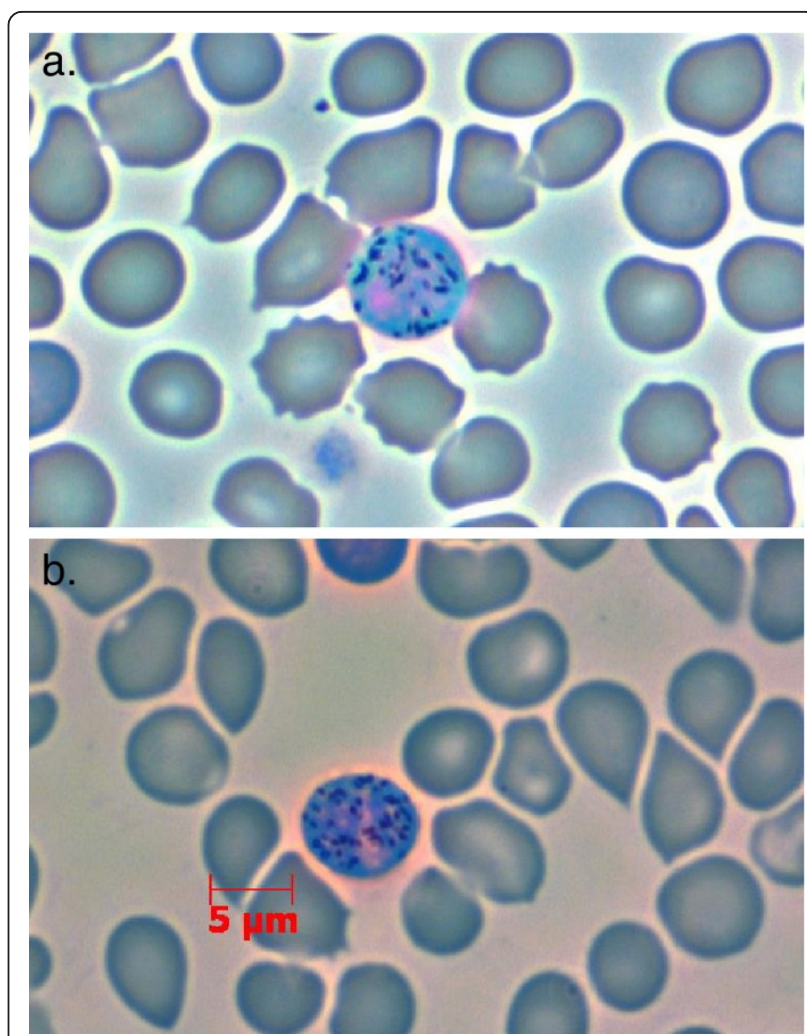

Figure 1 Polychromophilus murinus and $P$. melanipherus gametocytes. a. Polychromophilus murinus infection isolated from blood of a Daubenton's bat; b. P. melanipherus infection isolated from blood of a Schreibers' bent-winged bat. (Thin blood film, Giemsa-staining, phase contrast image).

hypotheses on its phylogenetic relationships: 1) it is monophyletic with the mammalian Plasmodium/Hepatocystis clade with which it shares the vertebrate host type, or 2) it shares its most recent common ancestor with the subgenus Haemoproteus (Haemoproteus), which has a similar vector. The genus Haemoproteus contains two avian subgenera which have different vectors. $H$. (Parahaemoproteus) spp. use biting midges as vectors, and $H$. (Haemoproteus) spp. are transmitted by Hippoboscidae, whose closest relatives are the bat flies [17]. A phylogeny based on ultrastructure and life-history traits grouped Polychromophilus together with both subgenera of Haemoproteus [8]. However, two recent molecular phylogenetic studies based on part of the cytochrome b sequence both suggest, despite their different topologies, a close relationship between Polychromophilus and sauropsid Plasmodium $[18,19]$. This fact provides a third hypothesis: 3) Polychromophilus is monophyletic with sauropsid Plasmodium (see Figure 2).

The aim of this study was to test these three hypotheses against each other. Though previous studies on the phylogenetic relationships of Polychromophilus have been done, all used only a single gene. Different genes in 


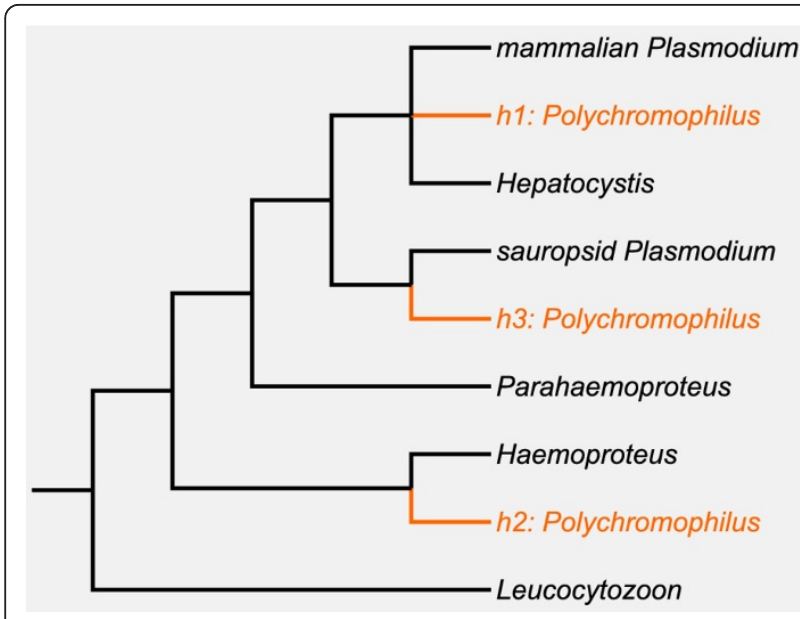

Figure 2 The hypothetical phylogeny of the genus Polychromophilus and the other Haemosporida. The hypothetical branches are marked in orange and based either on the conservation of the vertebrate host (hypothesis 1), the conservation of the dipteran vector (hypothesis 2), or based on previous molecular studies of the cytb gene (hypothesis 3 ).

a single organism can show different evolutionary patterns and it is, therefore, recommended to use multiple genes for accurate relationship estimation [20]. The four genes from three different genomes sequenced for this study represent two species of Polychromophilus (being the two type species of the two proposed subgenera). These sequences were subsequently combined with an existing dataset of 60 species of Haemosporida to clarify the phylogenetic relationships and gain insight into the evolutionary host switches of Polychromophilus.

\section{Methods}

\section{Sample collection and preparation}

Four Miniopterus schreibersii (Schreibers' bent-winged bat) were caught using mist nets in the entrance of an abandoned mine in western Switzerland under authorization 2203 issued by the Veterinarian Service of Canton Vaud, Switzerland. Blood was obtained by puncturing the uropatagial vein with a $0.5 \mathrm{~mm}$ gauge needle (Neolus). The blood beads that consequently formed on the uropatagium (between 10 and $30 \mu \mathrm{l}$ total) were taken up in a microvette with EDTA (Sarstedt) and stored at $4{ }^{\circ} \mathrm{C}$ until further analysis. Haemostatic cotton was applied on the punctured vein until the bleeding had stopped before releasing any bats.

One drop of blood was applied to a glass microscope slide for later visual identification of the parasite species. After smearing the blood, the slide was dried and immediately submerged in $100 \%$ methanol for fixation. Finally $5 \%$ Giemsa-staining was applied for one hour to stain the cells. DNA was extracted from whole blood using the DNeasy Blood and Tissue kit (Qiagen). Megali et al. [18] provided extracted DNA samples from blood of Myotis daubentoni (Daubenton's bat) which contained P. murinus infections. These infections were previously shown to be characterized by different cytochrome b haplotypes [18].

\section{Molecular analysis}

For the phylogenetic reconstruction, four genes were selected from the three cellular genomes: two mitochondrial DNA sequences, cytochrome b (cytb, $607 \mathrm{bp})$ and cytochrome oxidase subunit I (coI, $768 \mathrm{bp}$ ); one DNA sequence from the apicoplast, caseinolytic protease $\mathrm{C}$ (clpc, $502 \mathrm{bp}$ ); and one nuclear DNA sequence, adenylosuccinate lyase (asl, $186 \mathrm{bp}$ ).

All primer pairs used for the polymerase chain reactions were taken from Martinsen et al. [6] with the exception of coI nested Po, which was designed during this study (see Table 1 for primer sequences). All reactions started with an initial denaturation phase at $94^{\circ} \mathrm{C}$ for four minutes and one minute for the first and nested PCRs, respectively. The reactions ended with an annealing phase at $72^{\circ} \mathrm{C}$ for $7 \mathrm{~min}$. All cycles started for $30 \mathrm{~s}$ at $94^{\circ} \mathrm{C}$, but the other cycle conditions and the number of cycles differed depending on the primer pair used (see Table 1). The 25 $\mu \mathrm{l}$ reaction volume contained $3 \mu \mathrm{l}$ of extraction product, $0.25 \mathrm{U}$ Taq polymerase, $0.3 \mathrm{mM}$ of both primers, 0.25 mM dNTP's, 1× Qiagen PCR buffer and a total of $2 \mathrm{mM}$ $\mathrm{MgCl}$ (except for the reactions with the coI primers, which had a total of $3 \mathrm{mM} \mathrm{MgCl}$ ). The nested PCR reaction volume was similar except for the extraction product, which was replaced with $1 \mu \mathrm{l}$ of product of the first PCR. For the asl amplification the first PCR product was purified, which resulted in a better performance of the nested reaction.

All successfully amplified samples were purified according to the manufacturer's protocol using the Wizard PCR Clean-Up system (Promega) or the Minelute PCR Purification kit (Qiagen) in the case of asl. DNA concentrations were estimated by visualization on a $1.5 \%$ agarose gel with a $100 \mathrm{bp}$ reference ladder (Roche). For the sequencing reactions $\sim 20 \mathrm{ng}$ of purified PCR product, $2 \mu \mathrm{l}$ Big Dye Terminator v3.1 and $1 \mu \mathrm{l}$ of $10 \mathrm{mM}$ primer were mixed to a $10 \mu \mathrm{l}$ volume. Sequence analysis was performed on an ABI Prism 3,100 genetic analyser (Applied Biosystems). Sequence chromatographs were checked for ambiguities with Chromas Lite v2.01 (Technelysium).

\section{Phylogenetic reconstruction}

The obtained sequence data were combined with the same gene sequences of 60 other haemosporidian species obtained from GenBank (see Additional file 1). These 60 species represent the major clades of the Haemosporida, i.e. Leucocytozoon, Haemoproteus (Haemoproteus), 
Table 1 Name, sequence and PCR conditions of the primers used

\begin{tabular}{|c|c|c|c|c|c|}
\hline Name & & Primer sequence & Annealing & Extension & Cycles \\
\hline \multirow[t]{2}{*}{ asl/outer } & fw & GSKAARTTTAATGGKGCTGTWGG & $47^{\circ} \mathrm{C}, 30 \mathrm{~s}$ & $72^{\circ} \mathrm{C}, 50 \mathrm{~s}$ & 35 \\
\hline & $\mathrm{rv}$ & GGATTAAYTTTATGAGGCATTG & & & \\
\hline \multirow[t]{2}{*}{ asl/nested } & fw & GCTGATMAAAATRTTGATTGG & $50^{\circ} \mathrm{C}, 30 \mathrm{~s}$ & $72^{\circ} \mathrm{C}, 30 \mathrm{~s}$ & 38 \\
\hline & $\mathrm{rv}$ & GAGGCATTGTACTACTWCC & & & \\
\hline \multirow[t]{2}{*}{ clpc/outer } & fw & AAACTGAATTAGCAAAAATATTA & $50^{\circ} \mathrm{C}, 30 \mathrm{~s}$ & $72^{\circ} \mathrm{C}, 50 \mathrm{~s}$ & 38 \\
\hline & $r v$ & CGWGCWCCATATAAAGGAT & & & \\
\hline \multirow[t]{2}{*}{ clpc/nested } & fw & GATTTGATATGAGTGAATATATGG & $48^{\circ} \mathrm{C}, 30 \mathrm{~s}$ & $72^{\circ} \mathrm{C}, 30 \mathrm{~s}$ & 40 \\
\hline & $\mathrm{rv}$ & CCATATAAAGGATTATAWG & & & \\
\hline \multirow[t]{2}{*}{ col/outer } & fw & CTATTTATGGTTTCATTTTATTTGGTA & $57^{\circ} \mathrm{C}, 30 \mathrm{~s}$ & $72^{\circ} \mathrm{C}, 50 \mathrm{~s}$ & 35 \\
\hline & $\mathrm{rv}$ & AGGAATACGTCTAGGCATTACATTAAATCC & & & \\
\hline \multirow[t]{2}{*}{$\mathrm{col} /$ nested Po } & fw & AGCAATATCAATAGCTGCATTACCT & $62^{\circ} \mathrm{C}, 30 \mathrm{~s}$ & $72^{\circ} \mathrm{C}, 50 \mathrm{~s}$ & 38 \\
\hline & $\mathrm{rv}$ & GATTTCTTCAATATAATGCCTGGA & & & \\
\hline \multirow[t]{2}{*}{ cytb/outer } & fw & TAATGCCTAGACGTATTCCTGATTATCCAG & $55^{\circ} \mathrm{C}, 30 \mathrm{~s}$ & $72^{\circ} \mathrm{C}, 50 \mathrm{~s}$ & 35 \\
\hline & $\mathrm{rv}$ & TGTTTGCTTGGGAGCTGTAATCATAATGTG & & & \\
\hline \multirow[t]{2}{*}{ cytb/nested } & fw & TCAACAATGACTTTATTTGG & $55^{\circ} \mathrm{C}, 30 \mathrm{~s}$ & $72^{\circ} \mathrm{C}, 50 \mathrm{~s}$ & 40 \\
\hline & rv & TGCTGTATCATACCCTAAAG & & & \\
\hline
\end{tabular}

All denaturation and final extension periods are the same for all primer-pairs.

Haemoproteus (Parahaemoproteus), Hepatocystis and Plasmodium (including mammalian, avian and saurian). Sequences were aligned with ClustalW as implemented in MEGA version 5 [21]. The single-gene alignments were concatenated using FASconCAT [22].

All phylogenetic reconstructions were done using both Maximum Likelihood (ML) analysis and Bayesian inference (BI). For ML analysis, the PhyML software [23] was used for the single-gene alignments. Since PhyML does not allow for partitioning of the data RAxML [24] was used for the concatenated alignment. Models of nucleotide substitution were GTR $+\Gamma+\mathrm{I}$ for $c y t b, c o 1$ and $c l p c$ and $\mathrm{GTR}+\Gamma$ for $a s l$, as determined by MrAIC [25]. For each analysis, the transition rates of the GTR model, the shape of the $\Gamma$-distribution and the proportion of invariable sites were estimated by the program. Both the RAxML and PhyML analyses were assessed by performing 1,000 bootstrap replicates.

For the Bayesian analysis, the same models of character evolution as described for the ML analyses were implemented with MrBayes 3.1.2 [26]. In the concatenated analysis the data were again partitioned by gene, where each partition had its corresponding model and independent parameter estimations. The MCMC algorithm was done with four chains and was run for 20,000,000 generations, sampling every 1,000 generations. Two independent runs were performed to assess convergence to the correct posterior distribution. All parameters were checked for convergence using Tracer v1.5 and the first $10 \%$ of samples of each run was discarded as burn-in. All computations were performed on the Vital-IT cluster of the Swiss Institute of Bioinformatics.

\section{Rooting the tree}

Which outgroup to use has been a matter of debate lately. Perkins and Schall [7] identified Leucocytozoon as the most primitive clade of the order, using Theileria as an outgroup in their analysis of $c y t b$ sequences. But a recent study by Outlaw and Ricklefs [19] demonstrated that, when using a relaxed molecular clock, Leucocytozoon becomes the most derived group, effectively turning the tree inside-out. The authors argue that most ancient divergence should be between the mammal-infecting Plasmodium and Hepatocystis on the one side, and avian/saurian Plasmodium, both subgenera of Haemoproteus and Leucocytozoon on the other.

For the phylogenetic tree reconstructions, the Leucocytozoon spp. were initially selected as the outgroup, but these results were tested for their robustness by redoing the analyses using different rooting methods: 1) forcing the mammalian Plasmodium/Hepatocystis clade as outgroup instead of Leucocytozoon; 2) adding amino acid sequences of the more distantly related Babesia spp. as the outgroup (see Additional file 1) and repeating the ML analyses; 3) using the molecular clock methods similar to Outlaw and Ricklefs [19] but with varying priors: a Yule or birth-death tree prior, a strict, a log-normal relaxed or an exponential relaxed clock with a GTR $+\Gamma+$ I substitution model, 20 million generations sampling every 2,000 generations and two independent MCMC runs using BEAST [27-29].

\section{Topological tests}

The obtained Bayesian majority rule consensus tree was compared with each of the four Bayesian single-gene 
majority rule consensus trees to rule out any conflict in topology. The Kishino-Hasegawa tests [30] were performed in Treefinder [31]. The tests proved non-significant for all genes but asl (see Table 2). This gene was, therefore, removed from the concatenated alignment and a new phylogenetic reconstruction was performed on the remaining genes only.

For each of the three hypotheses on the Polychromophilus origin a corresponding topology was constructed. This was done by restricting the placing of Polychromophilus during tree reconstruction in RAxML, forcing it either with the mammal-infecting Plasmodium/Hepatocystis clade (hypothesis 1), the Haemoproteus (Haemoproteus) clade (hypothesis 2) or with the sauropsid Plasmodium clade (hypothesis 3 ). These restricted topologies were then tested together with the topology produced by the maximum likelihood analysis using a Shimodaira-Hasegawa test [32] as implemented in PAML 4 [33].

\section{Results}

The stained slides showed erythrocytes infected with slightly oval-shaped gametocytes (see Figure 1b). The granular appearance and pinkish staining at the nucleus fit the description of Polychromophilus melanipherus as given by Garnham [1]. The morphology of the observed gametocytes could therefore be linked to the molecular sequences obtained from the infections (Table 3).

None of the topologies obtained by independent analyses of the separate genes conflicted with the topology resulting from the concatenated alignment (KishinoHasegawa tests: $c y t b: \Delta \operatorname{lnl}=2.8, \mathrm{p}_{\mathrm{KH}}=0.432, c o I: \Delta \operatorname{lnl}=$ 12.1, $\left.\mathrm{p}_{\mathrm{KH}}=0.234, c l p c: \Delta \operatorname{lnl}=9.3, \mathrm{p}_{\mathrm{KH}}=0.270\right)$, except for asl $\left(\Delta \operatorname{lnl}=81.0, \mathrm{p}_{\mathrm{KH}}<0.001\right)$. Despite this strong rejection, both the ML and BI trees of asl had only few supported nodes and only closely related pairs were recovered (data not shown). A possible cause of the incongruence detected could be positive selection events in the evolution of the asl nuclear sequence [34]. However, analyses performed with Codeml [33] did not show signs of positive selection on the nuclear gene.

Table 2 The Kishino-Hasegawa topological test results

\begin{tabular}{llll} 
& Single gene tree & \multicolumn{2}{l}{ Concatenated 4 genes tree } \\
& InL & InL & pKH \\
\hline asl & -3561.406 & -3642.361 & $<0.001^{*}$ \\
clpc & -7718.852 & -7728.157 & 0.2696 \\
col & -10062.16 & -10074.33 & 0.2336 \\
cytb & -6856.617 & -6859.401 & 0.4323 \\
\hline
\end{tabular}

For each gene the likelihood of the phylogeny of that gene was compared to the phylogenetic reconstruction based on all four genes. The log-likelihood values and $\mathrm{p}$-values are shown per gene alignment. Only the asl alignment gives a significantly worse likelihood value for the tree based on the combined data, which indicates conflicting topologies.
Although the reasons for this DNA region to be rejected by the topology tests are unclear, the length of the asl gene fragment sequenced in this study is very small (186 $\mathrm{bp})$. This could suggest that random errors are responsible for creating the incongruences observed with this gene. Adding other, and especially longer, nuclear genes would certainly bring more information to test if the evolutionary relationships estimated from the different genomes are congruent or if specific gene trees best represent the evolution of each DNA regions. Different cellular genomes often have different evolutionary histories; even within a single genome not all genes show the same phylogenetic relationships [20].

Figure 3 presents the reconstructed phylogenetic trees using the combined data of $c y t b, c o I$ and $c l p c$ by ML and BI. The analyses produce no conflict on any of the major nodes. All major genera and subgenera are recovered and represented in the phylogenetic tree by separate monophyletic clades, with the exception of the sauropsid Plasmodium clade, which contains Polychromophilus within it.

\section{Diversity of Polychromophilus species}

Polychromophilus forms its own clearly defined clade in both the $\mathrm{BI}$ and $\mathrm{ML}$ reconstructions. Within this clade, the two species of Polychromophilus form well supported separate sister clades (see Figure 3). The distinction between $P$. melanipherus and $P$. murinus has often been made based on host species, since $P$. melanipherus was by definition confined to Miniopterus schreibersii as hosts. This distinction, however, has been qualified as 'arbitrary' and 'unsatisfactory' [1]. This study demonstrates for the first time that there is a clear genetic distinction between the two Polychromophilus species, confirming their taxonomic status of different species from a molecular point of view.

However, to determine whether this level of genetic divergence between $P$. murinus and $P$. melanipherus merits their placement in different subgenera [10] or even different genera [11], other species of the genus should be added (e.g. P. deanei [35] and P. adami [10]). Without these supplementary species, the overall observed genetic diversity within the genus Polychromophilus is low; it is clearly less than that of the genera Plasmodium and Haemoproteus or even less than the diversity found in subgenera like P. (Vinckeia) and H. (Parahaemoproteus). No critical level of genetic diversity exists as a precondition for the elevation of a subgenus, but the low diversity found within Polychromophilus does suggest that confirming $P$. (Bioccala) as a separate genus would cause a taxonomic asymmetry within the Haemosporida.

Two more haemosporidian genera infecting bats have been described: Dionisia [36] and Biguetiella [11]. Both contain only a single species and are described as 'little different' from Polychromophilus (Polychromophilus) [36] and 
Table 3 The haplotypes and corresponding accession numbers for GenBank per sequenced sample per gene

\begin{tabular}{|c|c|c|c|c|c|c|c|c|}
\hline & asl & & clpc & & $\mathrm{col}$ & & cytb & \\
\hline ind. & ht. & $a c c . n b$. & ht. & $a c c . n b$. & ht. & $a c c . n b$. & ht. & $a c c . n b$. \\
\hline 104 & Pmu1 & JN990725 & Pmu1 & JN990723 & Pmu1 & JN990718 & Pmu1 & JN990712 \\
\hline 114 & Pmu1 & .. & Pmu2 & JN990724 & - & - & Pmu1 & .. \\
\hline 156 & Pmu1 &. & Pmu1 &.. & Pmu2 & JN990719 & Pmu2 & JN990713 \\
\hline A2111 & - & & Pme3 & JN990720 & Pme3 & JN990714 & Pme3 & JN990708 \\
\hline A2112 & Pme2 & JN990726 & Pme4 & JN990721 & Pme4 & JN990715 & Pme4 & JN990709 \\
\hline A2113 & - & - & Pme5 & JN990722 & Pme5 & JN990716 & Pme5 & JN990710 \\
\hline A2114 & - & - & - & - & Pme6 & JN990717 & Pme6 & JN990711 \\
\hline
\end{tabular}

Samples 104, 114 and 156 are Polychromophilus murinus, sampled from Myotis daubentoni and shared some haplotypes. The samples A2111-A2114 are Polychromophilus melanipherus from Miniopterus schreibersii and never shared haplotypes. For each unique haplotype, the GenBank accession number is mentioned only once in the table. '..': accession number already mentioned. ' - ': sequencing was unsuccessful.

as 'a vicariant form of' Polychromophilus (Bioccala) [11], respectively. Whether their similarities to Polychromophilus spp. are because of convergence or shared ancestry can only be tested by combining the morphological data with molecular methods [37]. A big obstacle in studying these unfamiliar species however is the lack of observations. No other records of Biguetiella or Dionisia exist. Single descriptions of new parasite species found in a limited number of hosts are a problem encountered more often by parasitologists and can severely hamper classification [37].

\section{Polychromophilus' placement in the phylogeny of}

\section{Haemosporida}

The bootstrap value (69/100) suggests that the Polychromophilus clade is restricted to the Plasmodium branch of the haemosporidian tree. Even though this node also appears in the Bayesian majority rule consensus tree, the support for it is actually very weak (posterior probability = 0.73). However, the alternative hypothesis 2 , that Polychromophilus shares its most recent common ancestor with the subgenus Haemoproteus (Haemoproteus), is clearly rejected (Shimodaira-Hasegawa test; see Table 4).

It is less clear where within the Plasmodium clade Polychromophilus belongs. Neither phylogenetic method indicates that Polychromophilus originated from mammalian Plasmodium/Hepatocystis and both instead produced topologies suggesting a sauropsid origin (see Figure 3). However, the actual support for the node separating the mammalian clade from sauropsid Plasmodium/Polychromophilus clade is low. The BI supports the monophyly of

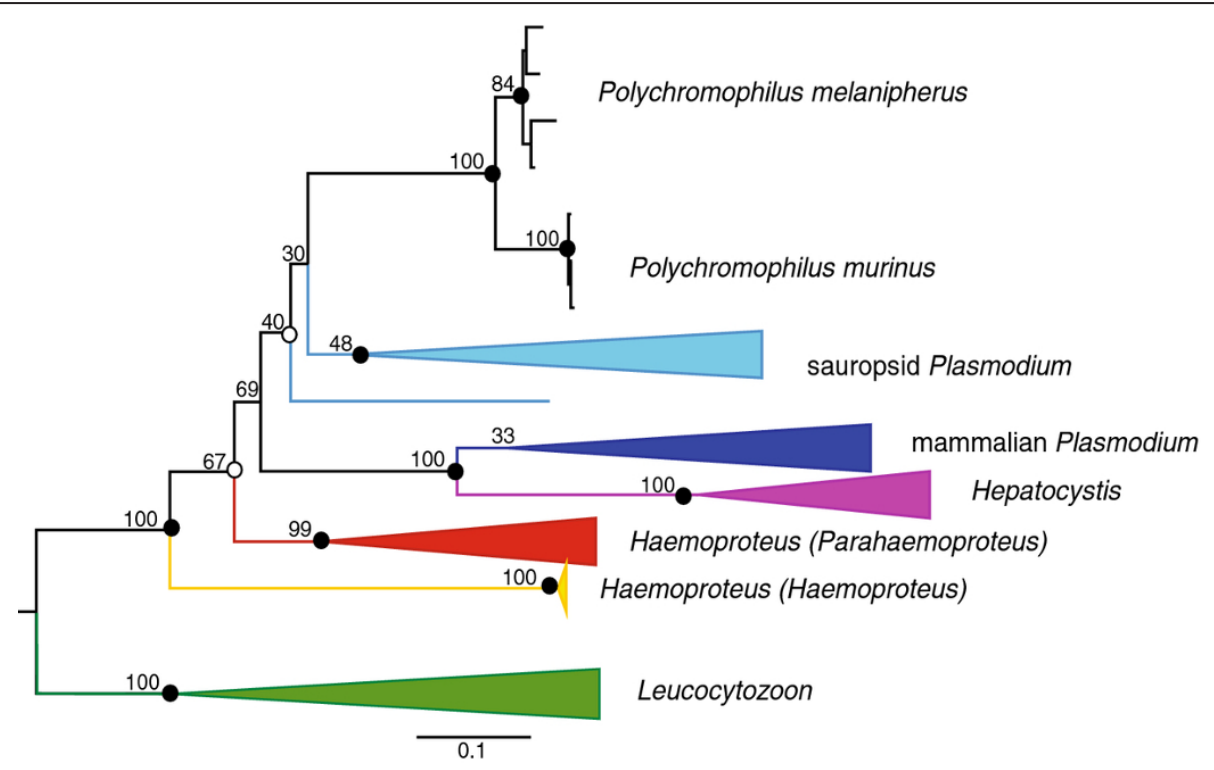

Figure 3 Polychromophilus shares its most recent common ancestor with avian and reptilian Plasmodium. Shown is the $50 \%$ majorityrule consensus tree from the Bayesian inference analysis. The phylogenetic reconstruction using maximum likelihood produced a similar tree. For clarity all clades except the Polychromophilus are collapsed and replaced by coloured triangles. Each colour represents a different haemosporidian group. The dots indicate Bayesian node support. Closed dots indicate a posterior probability $\geq 0.95$, open dots a posterior probability $\geq 0.90$. Node values indicate bootstrap values. Branch lengths represent the number of substitutions. The single blue branch belongs to a Plasmodium sp. infecting the skink Egernia stokesii. 
Table 4 The Shimodaira-Hasegawa topological test results comparing the three hypothetical topologies

\begin{tabular}{lll}
\hline Tree & $\boldsymbol{I n L}$ & $\boldsymbol{p}_{\boldsymbol{S H}}$ \\
\hline Best tree (hypothesis 3) & -25126.753 & - \\
Hypothesis 1 & -25130.147 & 0.578 \\
Hypothesis 2 & -25154.740 & $0.023^{*}$ \\
\hline
\end{tabular}

The best tree was the tree provided by the maximum likelihood analysis (see Figure 3) and concurred with hypothesis 3 . The log-likelihoods of the other two trees, based on hypothesis 1 and 2 (see Figure 2), are compared with the best tree. The hypothesis 2 tree, which has Polychromophilus grouped with Haemoproteus, has a significantly worse fit and can be rejected.

sauropsid Plasmodium and Polychromophilus (hypothesis 3 ) with a posterior probability of 0.92 , but the ML support of that same critical node is absent (bootstrap value of 40/100). The topological test comparing the different phylogenetic scenarios did not provide more support for either hypothesis 1 or 3 (see Table 4).

Most of the alternative rooting methods favour hypothesis 3. Indeed, rooting the tree with the mammalian Plasmodium clade instead of Leucocytozoon, as suggested by Outlaw and Ricklefs [19], validates the conclusion of a sauropsid origin of Polychromophilus in both BI and ML (see Additional file 2).

The choice of the prior distributions guiding either the distribution of mutation rates across the tree (log-normal vs exponential) or the divergence times (Yule vs birthdeath) does not change the conclusion. All the molecular clock analyses place Polychromophilus within the sauropsid Plasmodium clade, with clade credibilities between 0.87 and 1 . However the root itself does change depending on the prior set. The Yule and log-normal prior lead to the placement of the Leucocytozoon as the outgroup, whereas the mammalian Plasmodium/Hepatocystis clade is placed as the outgroup with the birth-death tree and exponential relaxed clock (see Additional file 2).

The ML analysis using Babesia as an outgroup produces a topology with very little support. All major nodes have bootstrap values of $<50 / 100$, so no outgroup can be identified, nor can Polychromophilus be placed within the tree with any confidence (see Additional file 3). The genetic divergence between Babesia (Piroplasmida) and the Haemosporida is very high, which results in a very long branch leading to the Babesia lineages. This changes the rooting procedure to a problem of 'long-branch attraction' with all corresponding biases [38], and these analyses should therefore be approached with caution [19].

None of the used phylogenetic methods reject our third hypothesis, stating that Polychromophilus is monophyletic with the sauropsid Plasmodium clade. ML and the topological test could not discriminate between hypothesis 2 and 3, but BI and molecular clock rooting methods gave more support for the latter hypothesis.
These analyses are far from conclusive, but do suggest that Polychromophilus did not evolve from a mammalinfecting ancestor, but has instead invaded the mammalian class of hosts independently.

Our results show that the three DNA regions used in the combined matrix do not provide sufficient phylogenetic information to unambiguously place the Polychromophilus lineage. When combining regions from different genomes, this could introduce sufficient conflict to reduce the confidence in the reconstructed trees, even if the topology tests did not identified major incongruence. The way forward to clearly place the Polychromophilus lineage within the large Plasmodium clade is to sequence longer stretches of DNA regions, in particular from the nuclear genome, and to use gene tree approaches to identify the best evolutionary relationships at the species level $[39,40]$.

\section{Previous findings}

The close relation between Polychromophilus and avian Haemosporida has been suggested before. Carreno et al. [8] produced a phylogeny based on life-history and ultrastructure characters and concluded that Polychromophilus is most closely related to Haemoproteus, a hypothesis rejected by the current study. Megali et al. [18] used a 705 bp cytb fragment and concluded that Polychromophilus shared its closest common ancestry with avian Plasmodium. However, the base of their tree was not well resolved. The authors themselves recommended the use of multiple genes.

Duval et al. [41] discussed bat Haemosporida, but never identified the species. However, their molecular analyses, again using only $c y t b$, grouped their samples clearly with sauropsid Plasmodium, leading to a similar conclusion as the current study. In their paper, they cautiously did not name their collected species. However, the corresponding sequences that are available in GenBank have been identified as 'Hepatocystis sp.'. Based on the work of Megali et al. [18] it is very likely that part of those sequences are actually Polychromophilus species. Misidentification is a big obstacle in apicomplexan research as a whole [42] and haemosporidian research in particular [43]. Therefore, caution is required when naming species for GenBank.

\section{Switch of host, switch of vector}

Parasitizing a new, mammalian host likely necessitated many adaptive changes, given their characteristic, nonnucleated red blood cells. The $c y t b$ DNA region sequenced here showed long branches of non-synonymous substitutions separating the avian from the mammal clade [4]. Many lineages have become extinct over time during the evolution towards the mammalian and avian Plasmodium lineages [44]. Nevertheless Polychromophilus' origin suggests that the switch to mammalian hosts happened at least twice during Haemosporida evolution. Rayella is 
thought to have originated from Hepatocystis [45] and has been classified as such [1], but Nycteria's origins are more elusive; whether it is a case of yet another independent host switch, or an ancient mammalian Plasmodium lineage that has survived the pruning on that branch, remains to be investigated.

Haematophagy has appeared multiple times in the evolution of the Diptera. It evolved once at the origin of the superfamily Hippoboscoidea and is shared by all its members [17]. Consequently, many Hippoboscoidea spp. are implicated in the transmission of diseases, most notably sleeping sickness (Glossinidae) and malaria (Hippoboscidae and Nycteribiidae). The relatively high relatedness of the latter two families [17] is not reflected by their haemosporidian parasites. This study convincingly rejected the hypothesis that hippoboscid-transmitted H. (Haemoproteus) shares its most recent common ancestor with the nycteribid-transmitted Polychromophilus. A cospeciation event of these Haemosporida with their dipteran hosts can, therefore, clearly be excluded.

Instead, Polychromophilus' ancestor must have been vectored by of a member of the Culicidae, as are all modern Plasmodium species. Culicidae are one of the oldest members of the Diptera, an order with a higher radiation of species than all terrestrial vertebrates put together [46]. The phylogenetic distance between Culicidae and Nycteribiidae is one of the largest within the order [46], yet the adaptations required for this new vector were seemingly acquired in parallel to those required for the new mammalian host.

Because the Nycteribiidae are completely specialised to bats, the first appearance of Polychromophilus in bats must have been mediated by either mosquitoes or via the hippoboscid flies. Many Culicidae spp. feed on both mammals and birds readily, and within the Hippoboscidae, the host switch from mammals to birds has happened several times [17]. Therefore, both could have been responsible for the first transmission. However, once Polychromophilus' ancestor was introduced in bats, adapting to the nycteribid vectors likely had large fitness advantages. Specifically, the haematophagous lifestyle of both males and females combined with their high prevalence on bats [13], and ease of moving between bat-hosts (unpublished observations), make the Nycteribiidae an ideal vector for the protozoan parasite. However, this same switch to Nycteribiidae also limited the potential range of Polychromophilus vertebrate hosts to the Chiroptera.

\section{Conclusions}

The phylogenetic reconstruction of three genes of Polychromophilus spp. demonstrates that the P. melanipherus and $P$. murinus are clearly two genetically distinct species. Only the addition of the other Polychromophilus spp. can validate the current division of Polychromophilus in separate subgenera. Polychromophilus is clearly not related to Haemoproteus (Haemoproteus). Instead Bayesian inference and molecular clock outgroup free phylogenetic reconstructions suggest that the Polychromophilus most likely had a birdor reptile-infecting Plasmodium ancestor. The switch to mammalian hosts would, therefore, not have occurred once, but at least twice in the haemosporidian evolutionary past. This event was accompanied by the adaptation to a new, phylogenetically distant dipteran vector.

\section{Additional material}

\section{Additional file 1: Species name, host and accession numbers of sequences retrieved from GenBank for the phylogenetic reconstructions. This table contains additional host information and the GenBank accession numbers of all genes used for the phylogenetic analyses. Not all gene sequences are available for all species, missing sequences are denoted by '-'.}

Additional file 2: Changing topologies for alternative rooting methods. Changing topologies acquired by different methods of phylogenetic reconstruction. Irrespective of the root, Polychromophilus remains nested within the sauropsid Plasmodium clade. a. The original best tree from maximum likelihood reconstruction, but now rooted with the mammalian Plasmodium/Hepatocystis, as suggested by Outlaw and Ricklefs [16]. Topologies b. and c. are acquired using a relaxed molecular clock with no predefined root, GTR $+\Gamma+$ I substitution model, 20 million generations sampling every 2,000 generations and two independent MCMC runs using BEAST. All nodes have clade credibilities $>0.5 \mathrm{~b}$. Topology acquired with the Yule tree prior and an exponential relaxed clock. c. Topology acquired with the birth-death tree prior and a log-normal relaxed clock. The different haemosporidian clades are represented by the coloured triangles. The clade height represents the number of containing taxa.

Additional file 3: A topology rooted with Babesia provides little information. The amino acid alignment provides too little contrast to construct a tree with high support as most nodes are unsupported. A very long branch separates the Babesia species from all Haemosporida. Shown is the best tree of a ML analysis using a $J T T+\Gamma+$ I substitution model and bootstrapping a 1,000 times. Closed dots: bootstrap value > 90; Open dots: bootstrap values $>50$.

\section{Acknowledgements}

We would like to give a big thank you to Anne-Lyse Ducrest and Céline Simon for advice on and assistance with the molecular work. We would also like to give our gratitude to Emily Clark and two anonymous referees for helpful comments improving this manuscript. We thank the Swiss Institute of Bioinformatics for their support and the availability of the Vital-IT facilities. This research was supported by grant 31003A_120479 from the Swiss National Science Foundation.

\section{Author details}

'Département d'Ecologie et Evolution, Université de Lausanne, Biophore, UNIL-Sorge, 1015 Lausanne, Switzerland. 'Swiss Institute of Bioinformatics, Quartier Sorge, 1015 Lausanne, Switzerland.

\section{Authors' contributions}

FW collected the samples, carried out the molecular work, performed the phylogenetic analyses and drafted the manuscript. NS participated in the phylogenetic analyses. PC conceived the study, collected the samples and interpreted the results. All authors read and approved the final manuscript.

\section{Competing interests}

The authors declare that they have no competing interests. 
Received: 21 November 2011 Accepted: 22 February 2012

Published: 22 February 2012

\section{References}

1. Garnham PCC: Malaria Parasites and Other Haemosporidia. 1 edition. Oxford: Blackwell Scientific Publications; 1966.

2. Dasgupta B: A new malarial parasite of the flying squirrel. Parasitology 1967, 57:467-474.

3. Valkiũnas G: Avian Malaria Parasites and Other Haemosporidia. Englishth edition. Boca Raton: CRC Press; 2005.

4. Outlaw DC, Ricklefs RE: Comparative gene evolution in haemosporidian (Apicomplexa) parasites of birds and mammals. Mol Biol Evol 2010, 27:537-542.

5. Yotoko KSC, Elisei C: Malaria parasites (Apicomplexa, Haematozoea) and their relationships with their hosts: is there an evolutionary cost for the specialization? J Zool Syst Evol Res 2006, 44:265-273.

6. Martinsen ES, Perkins SL, Schall JJ: A three-genome phylogeny of malaria parasites (Plasmodium and closely related genera): evolution of lifehistory traits and host switches. Mol Phylogenet Evol 2008, 47:261-273.

7. Perkins SL, Schall JJ: A molecular phylogeny of malarial parasites recovered from cytochrome b gene sequences. J Parasitol 2002, 88:972-978.

8. Carreno RA, Kissinger JC, McCutchan TF, Barta JR: Phylogenetic analysis of haemosporinid parasites (Apicomplexa: Haemosporina) and their coevolution with vectors and intermediate hosts. Arch Protistenkd 1997, 148:245-252.

9. Garnham PCC: The zoogeography of Polychromophilus. Ann Parasitol Hum Comp 1973, 48:231-242.

10. Landau I, Rosin G, Miltgen F, Hugot JP, Leger N, Beveridge I, Baccam D: Sur le genre Polychromophilus (Haemoproteidae, parasite de microchiroptères). Ann Parasitol Hum Comp 1980, 55:13-32.

11. Landau I, Baccam D, Ratanaworabhan N, Yenbutra S, Boulard Y, Chabaud AG: Nouveaux Haemoproteidae parasites de chiroptères en Thailande. Ann Parasitol Hum Comp 1984, 59:437-447.

12. Gardner RA, Molyneux DH: Polychromophilus murinus: a malarial parasite of bats: life-history and ultrastructural studies. Parasitology 1988, 96:591-605.

13. Dick CW, Patterson BD: Bat flies: obligate ectoparasites of bats. In Micromammals and Macroparasites: From Evolutionary Ecology to Management. Edited by: Morand S, Krasnov BR, Poulin R. Tokyo: SpringerVerlag; 2006:179-194

14. Theodor O: An Illustrated Catalogue of the Rothschild Collection of Nycteribiidae London: British Museum (Natural History); 1967.

15. Corradetti A: Alcuni protozoi parassiti di Nycteribiidae del genere Listropoda. Ann Igiene 1936, 46:444-460.

16. Mer GG, Goldblum N: A haemosporidian of bats. Nature 1947, 159:444-444.

17. Petersen FT, Meier R, Kutty SN, Wiegmann BM: The phylogeny and evolution of host choice in the Hippoboscoidea (Diptera) as reconstructed using four molecular markers. Mol Phylogenet Evol 2007 45:111-122.

18. Megali A, Yannic G, Christe P: Disease in the dark: molecular characterization of Polychromophilus murinus in temperate zone bats revealed a worldwide distribution of this malaria-like disease. Mol Ecol 2011, 20:1039-1048.

19. Outlaw DC, Ricklefs RE: Rerooting the evolutionary tree of malaria parasites. Proc Natl Acad Sci USA 2011, 108:13183-13187.

20. Cummings MP, Meyer A: Magic bullets and golden rules: data sampling in molecular phylogenetics. Zoology (Jena) 2005, 108:329-336.

21. Tamura K, Peterson D, Peterson N, Stecher G, Nei M, Kumar S: MEGA5: molecular evolutionary genetics analysis using maximum likelihood, evolutionary distance, and maximum parsimony methods. Mol Biol Evol 2011, 28:2731-2739.

22. Kück P, Meusemann K: In FASconCAT, version 1.0. Zool. Edited by: Forschungsmuseum A. Koenig, Germany; 2010:.

23. Guindon S, Gascuel O: A simple, fast, and accurate algorithm to estimate large phylogenies by maximum likelihood. Syst Biol 2003, 52:696-704.

24. Stamatakis A: RAxML-VI-HPC: maximum likelihood-based phylogenetic analyses with thousands of taxa and mixed models. Bioinformatics 2006, 22:2688-2690

25. Nylander JAA: MrAIC.pl. Uppsala. Program Distributed by the Author Evolutionary Biology Centre, Uppsala University; 2004
26. Ronquist F, Huelsenbeck JP: MrBayes 3: Bayesian phylogenetic inference under mixed models. Bioinformatics 2003, 19:1572-1574.

27. Drummond AJ, Ho SYW, Phillips MJ, Rambaut A: Relaxed phylogenetics and dating with confidence. PLOS Biol 2006, 4:699-710.

28. Drummond AJ, Nicholls GK, Rodrigo AG, Solomon W: Estimating mutation parameters, population history and genealogy simultaneously from temporally spaced sequence data. Genetics 2002, 161:1307-1320.

29. Drummond AJ, Rambaut A: BEAST: Bayesian evolutionary analysis by sampling trees. BMC Evol Biol 2007, 7:214

30. Kishino $H$, Hasegawa M: Evaluation of the maximum-likelihood estimate of the evolutionary tree topologies from DNA-sequence data, and the branching order in Hominoidea. J Mol Evol 1989, 29:170-179.

31. Jobb G: TREEFINDER Munich, Germany: Distributed by the author; 2008 [http://www.treefinder.de/], October 2008 edition.

32. Shimodaira H, Hasegawa M: Multiple comparisons of log-likelihoods with applications to phylogenetic inference. Mol Biol Evol 1999, 16:1114-1116.

33. Yang ZH: PAML 4: phylogenetic analysis by maximum likelihood. Mol Biol Evol 2007, 24:1586-1591.

34. Christin P-A, Besnard G, Edwards EJ, Salamin N: Effect of genetic convergence on phylogenetic inference. Mol Phylogenet Evol

35. Garnham PCC, Lainson R, Shaw JJ: A contribution to the study of Haematozoon parasites of bats - a new Haemoproteid, Polychromophilus deanei n. sp. Mem Inst Oswaldo Cruz 1971, 69:119-125.

36. Landau I, Chabaud AG, Miltgen F, Baccam D: Dionisia bunoi n. gen. n. sp. Haemoproteidae parasite du microchiroptère Hipposideros cyclops au Gabon. Ann Parasitol Hum Comp 1980, 55:271-280

37. Perkins SL, Martinsen ES, Falk BG: Do molecules matter more than morphology? Promises and pitfalls in parasites. Parasitology 2011, 138:1664-1674

38. Sanderson MJ, Shaffer HB: Troubleshooting molecular phylogenetic analyses. Annu Rev Ecol Syst 2002, 33:49-72.

39. Ane C, Larget B, Baum DA, Smith SD, Rokas A: Bayesian estimation of concordance among gene trees. Mol Biol Evol 2007, 24:412-426.

40. Heled J, Drummond AJ: Bayesian inference of species trees from multilocus data. Mol Biol Evol 2010, 27:570-580.

41. Duval L, Robert V, Csorba G, Hassanin A, Randrianarivelojosia M, Walston J, Nhim T, Goodman SM, Ariey F: Multiple host-switching of Haemosporidia parasites in bats. Malar J 2007, 6:157.

42. Morrison DA: Evolution of the Apicomplexa: where are we now? Trends Parasitol 2009, 25:375-382.

43. Valkiũnas G, Atkinson CT, Bensch S, Sehgal RNM, Ricklefs RE: Parasite misidentifications in GenBank: how to minimize their number? Trends Parasitol 2008, 24:247-248.

44. Ricklefs RE, Outlaw DC: A molecular clock for malaria parasites. Science 2010, 329:226-229.

45. Mattingly PF: The paleogeography of mosquito-borne disease. Biol J Linn Soc Lond 1983, 19:26.

46. Wiegmann BM, Trautwein MD, Winkler IS, Barr NB, Kim JW, Lambkin C, Bertone MA, Cassel BK, Bayless KM, Heimberg AM, Wheeler BM, Peterson KJ Pape T, Sinclair BJ, Skevington JH, Blagoderov V, Caravas J, Kutty SN, Schmidt-Ott U, Kampmeier GE, Thompson FC, Grimaldi DA, Beckenbach AT, Courtney GW, Friedrich M, Meier R, Yeates DK: Episodic radiations in the fly tree of life. Proc Natl Acad Sci USA 2011, 108:5690-5695.

doi:10.1186/1475-2875-11-53

Cite this article as: Witsenburg et al: The evolutionary host switches of Polychromophilus: a multi-gene phylogeny of the bat malaria genus suggests a second invasion of mammals by a haemosporidian parasite. Malaria Journal 2012 11:53 